\title{
TIPOS DE ORGANIZACIONES DEPORTIVAS EN ESPAÑA
}

\author{
TYPES OF SPORTS ORGANIZATIONS IN SPAIN
}

\author{
Paloma Escamilla-Fajardo ${ }^{1}$ \\ Mario Alguacil ${ }^{2}$ \\ Maria del Carmen Giménez-Espert ${ }^{3}$
}

\section{Resumen}

El deporte ha sido considerado un fenómeno de masas con un trascendental poder de seguimiento y congregación, aportando un importante valor económico y social. Hoy en día, en España nos encontramos en un entorno cambiante, en el que las demandas y necesidades de la población son cada vez más diversas, teniendo como consecuencia una obligatoria adaptación y versatilidad por parte de las organizaciones. Dichos cambios estructurales, funcionales y organizativos requieren de una sostenibilidad, tanto de las instalaciones deportivas como de los servicios ofertados. Este hecho cada vez se hace más complicado, por lo que el concepto tradicional de organización deportiva ha ido cambiando. En este estudio se han analizado los diferentes tipos de organizaciones en el mundo del deporte, englobadas en los tres ámbitos de actuación más importantes - ámbito público, privado y asociativo. Asimismo, dentro de cada ámbito encontramos diferentes instituciones y/o organizaciones muy diferentes. El objetivo del presente trabajo es analizar la tipología de organizaciones deportivas en España y su evolución en los últimos años.

\section{Palabras clave}

Organización deportiva, público, privado, asociativo, tipología.

\section{Abstract}

Sport has been considered a mass phenomenon with a trascendental power to follow and congregate, as well as its significant economic and social contribution. At the present time, in Spain we find ourselves in a changing environment, in which the demands and needs of the population are increasingly diverse, resulting in a mandatory adaptation and versatility on the part of organizations. These structural, functional and organizational changes require sustainability of both the sports facilities and the services offered. This is becoming more and more complicated, and the traditional concept of sports organization has changed. This study has analyzed the different types of organizations in the world of sport, encompassed in the three most important areas of action - public, private and associative. Also, within each field we find different institutions and/or organizations very different. The aim of this paper is to analyses the typology of sports organizations in Spain and their evolution in recent years.

\section{Keywords}

Sports organization, public, private, associative, typology.

\footnotetext{
${ }^{1}$ Universidad de Valencia (España). Correo electrónico: paloma.escamilla@uv.es.

2 Universidad Católica de Valencia (España). Correo electrónico: mario.alguacil@ucv.es.

${ }^{3}$ Universidad Europea de Valencia (España). Correo electrónico: carmen.gimenez@universidadeuropea.es.
} 


\section{Introducción}

La actividad física y el deporte son valiosas herramientas que aumentan la calidad de vida de las personas y cumplen una importante labor social (Leiva, Márquez, Rodríguez, Navas y Bozal, 2017). En su evolución reciente, han pasado de caracterizarse por unas políticas deportivas poco rigurosas y muy voluntaristas (que asumían como primer objetivo la satisfacción de demandas inmediatas y no tanto la creación de un proyecto deportivo general), a convertirse en un sector productivo de primera magnitud, según sostienen Gallardo, Guerrero y Gómez (2004).

Además de la creciente importancia económica, existe una evolución en la tendencia de la práctica deportiva en España, llegándose a catalogar como fenómeno social relevante a finales del siglo XX y comienzos del XXI. Según García Ferrando y Llopis Goig (2017), existe una popularización deportiva en los últimos años, llegándose a duplicar la práctica deportiva en la población con edades comprendidas entre 15 y 65 años desde 1980 hasta 2015. La tasa de práctica deportiva española pasa de un $25 \%$ en 1980 a un 53\% en 2015.

«El significativo aumento de la importancia social y económica del deporte en las últimas décadas, junto a la posición central y estratégica que ocupa en las sociedades modernas, por su contribución a la identidad individual y legitimidad política, integración social, relaciones internacionales, regeneración y desarrollo económico de la comunidad en la que se practica o en la que se realiza un evento deportivo, obliga a abandonar cualquier planteamiento que lo considere y aborde como una actividad o sector de carácter meramente marginal o secundario» (Sanz e Insúa, 2003, p. 78).

El impacto económico del deporte ha mejorado significativamente en estos últimos años (SalgadoBarandela, Barajas y Sánchez-Fernández, 2017), pero también lo ha hecho la valoración social. La práctica deportiva ha pasado de ser una actividad marginal y poco valorada por los ciudadanos, a formar parte del día a día en la vida de las personas.

Este desarrollo se ha sustentado en una estructura que, en España, y de acuerdo con la Ley 10/1990 del Deporte, se basa en un sistema de colaboración entre los sectores público, privado y asociativo. Los tres sectores tienen encomendadas responsabilidades en el fomento, promoción y desarrollo de las actividades y prácticas físico-deportivas. Estas responsabilidades, en muchos casos, han acabado convergiendo, solapándose y en ocasiones, confundiéndose. Por ello, el presente estudio tiene como objetivo analizar los tipos de organizaciones deportivas en España. Considerando de vital importancia tratar previamente las características fundamentales de los tres ámbitos deportivos de actuación (ámbito público, privado y asociativo).

\section{Ámbito deportivo público: Administración pública}

Elcuerpo público del deporte en España se estructura en los tres niveles de responsabilidad del sistema administrativo. Las funciones reguladoras de la Administración Central y las Administraciones Autonómicas, se trasladan a nivel operativo hasta el ciudadano a través de la función principalmente gestora y prestadora de servicios de la Administración local.

En primer lugar, dentro de la Administración Estatal, el Consejo Superior de Deportes (CSD), está adscrito al Ministerio de Educación, Cultura y Deporte, considerándose un organismo autónomo de carácter administrativo que actúa directamente en el cumplimiento de la normativa que establecen los poderes públicos.

Por otro lado, y teniendo en cuenta las comunidades autónomas, las direcciones generales de deportes de las comunidades autónomas están establecidas en los Estatutos de Autonomía, asegurando el cumplimiento de las normas reguladoras en el ámbito territorial autonómico correspondiente. Una de las acciones más importantes sería la coordinación de las Federaciones Deportivas comarcales, teniendo la potestad legislativa en cuanto a la política deportiva, programación de subvenciones, formación de técnicos deportivos y gestión de los centros formadores de Ciencias de la Actividad Física y del Deporte.

La Administración pública es el eje principal en la promoción y desarrollo de la práctica de actividad físico-deportiva desde hace muchos años (Fernández, Couto y Aguilar, 2015). Según Gallardo et al. (2004), con independencia del modelo de gestión del servicio, son tres las actuaciones más importantes que corresponden a la administración local: (i) la promoción del deporte entre todos los ciudadanos del municipio, mediante acciones que facilitan el conocimiento y acceso al deporte en 
general y a las instalaciones deportivas en particular; (ii) la construcción de instalaciones, centrándose más en la remodelación de las ya existentes que en construir nuevas y; (iii) el logro de la rentabilidad económica, intentando el mínimo gasto público, estudiando coste y eligiendo el tipo de gestión más adecuada para ofrecer servicios de la forma más eficaz.

Dentro de estos tres niveles de actuación, las entidades locales son las principales promotoras de servicios deportivos públicos, debido a dos hechos: el grado de proximidad al ciudadano y sus demandas, y una mejor posición de cara a la gestión de las instalaciones deportivas. Asimismo, la Administración local es la que más y mejor favorece y posibilita el desarrollo y fomento de la actividad deportiva a través de distintos tipos de estructuras (Servicios Deportivos Municipales, Patronatos, Fundaciones, Organismos Autónomos) y diferentes programas (Escuelas Deportivas Municipales, programas de fomento para distintos tipos de poblaciones, programas de colaboración y potenciación del asociacionismo deportivo, etc.).

Además de lo anterior, en cuanto a la materia deportiva se refiere, las entidades locales tienen alguna competencia más, destacando la reserva de suelo para la posible construcción de instalaciones deportivas, la colaboración y/o ayuda a los clubes deportivos en su territorio y la organización de competiciones locales.

Según el INE (2017) España cuenta con un total de 8.124 municipios, de los que un $84,01 \%$ tienen menos de 5.000 habitantes, el 11,04 entre 5.001 y 20.000 , el $4,19 \%$ entre 20.001 y 100.000 y el $0,76 \%$ más de 100.001 habitantes. Los municipios, casi sin exclusión, se pueden considerar como los mayores dinamizadores de la práctica deportiva en los últimos 30 años.

El gasto público vinculado al deporte en los municipios españoles ha sido, en los últimos 10 años, un reflejo del contexto de crisis que ha afectado a la sociedad y sus organizaciones (Conde-Ruiz, Díaz, \& Marín, 2016). Sin embargo, pese a los años de reducciones presupuestarias, en los últimos 4 años se puede observar una ligera tendencia positiva en el presupuesto del CSD para el deporte. Sirvan de ejemplo los datos recogidos de las Estadísticas de Liquidación de los Presupuestos, disponibles en el Ministerio de Hacienda y Función Pública (MHFP) y de las Cuentas Generales de cada Comunidad Autónoma, para el presupuesto liquidado de la Administración pública española, que arrojaban un significativo aumento del presupuesto destinado al fomento del deporte, que pasaba de un importe total de 1.903 millones de euros en 2013 a 1.964 millones en 2014; 2.115 millones en 2015 y 2.075 en 2016 (MECD, 2018, p. 71). Estos datos han ido mejorando, pero hay todavía mucho que acrecentar de los datos recogidos en 2011, donde se destinaron 2.501 millones de euros de gasto público al deporte.

Mención aparte merecen, para acabar de comprender las condiciones de prestación de servicios de los Ayuntamientos españoles, las fórmulas de gestión de los servicios deportivos públicos. El marco normativo español considera diversas posibilidades de prestación de los servicios, siempre bajo el carácter de «servicio público».

La prestación directa de servicios deportivos todavía sigue vigente en numerosos ayuntamientos, pero se está transformando en otros muchos. Este tradicional tipo de gestión deportiva municipal se caracteriza por realizar, coordinar y gestionar los servicios a través de personal propio y dependiendo completamente del presupuesto municipal. Entre las funciones más importantes en la gestión directa encontraríamos la administración de recursos disponibles y la oferta de servicios deportivos de forma eficaz, así como la coordinación y regulación del uso de instalaciones y espacios deportivos encontrando una gestión viable y sostenible.

Sin embargo, en un espacio corto de tiempo, la crisis ha llegado a dinamitar el modelo de empleo público, teniendo lugar un cambio en los tipos de gestión. Para poder garantizar los servicios de forma viable, es cada vez más factible el reclamo del sector privado en las administraciones públicas. Esta gestión indirecta, consecuencia de una externalización del servicio, está implantándose en el país de forma importante, intentando mejorar el control administrativo mediante un pliego de condiciones que puede dotar a la entidad externa de mayor o menor autonomía y control (Grimaldi, Fernández y García-Fernández, 2013). Esto quiere decir que no se cede el control y responsabilidad del servicio e instalaciones a su libre albedrío, sino que, según Calabuig, Quintanilla y Mundina (2008) son las propias administraciones públicas las que demandan a las organizaciones privadas, a las que se les adjudica la prestación de servicios, el cumplimiento de unos mínimos de calidad en la atención al ciudadano y eficacia en los servicios prestados.

Según Quesada (2014), las explotaciones deportivas 
a través de empresas concesionarias o sociedades mercantiles con distinta distribución en la propiedad, asumen el fomento y servicio del deporte, así como la protección y defensa de los intereses públicos. No obstante, cabe destacar que existen diferentes tipos de gestión indirecta, ya que los pliegos de condiciones pueden ser modificados basándose en un ajuste de los intereses de los municipios y las empresas implicadas. La administración pública tiene la obligación de controlar el cumplimiento de los compromisos del concesionario $\mathrm{y}$ todas las responsabilidades derivadas como la administración o el personal.

De una u otra forma, se pueden hallar en el panorama de la gestión municipal española la práctica totalidad de las combinaciones posibles de agentes gestores. A modo de ejemplo, el estudio de Arboledas y Puig (2012) hallaba una comparativa entre municipios andaluces y catalanes con una gran dispersión de modelos de gestión, si bien se observaba una tendencia en los municipios catalanes hacia una gestión compartida con el sector asociativo y privado; mientras que en Andalucía destacaba la oferta gestionada directamente por los Ayuntamientos. En este sentido, lo que parece apuntar las claras diferencias territoriales por comunidades, que abunda en la idea de una gran heterogeneidad a nivel estatal.

\section{Ámbito deportivo asociativo}

Como ya hemos citado anteriormente, el sistema deportivo se ha visto obligado a evolucionar en la misma línea que lo realizó, la diversidad de los practicantes en el panorama deportivo. Todo ello, lejos de significar un descenso de practicantes, lo que ha producido es una mayor heterogeneidad tanto en deportistas como en entidades. España posee una estructura en la que conviven diversidad de organizaciones y entidades deportivas (Rodríguez et al., 2018). Aunque a priori se relacione el ámbito asociativo con el tradicionalmente conocido club deportivo, si únicamente mencionásemos dicho tipo de organización dejaríamos fuera otros muy representativos, como las asociaciones o uniones deportivas.

Según el MECD (2017, p.102), los clubes deportivos son las «asociaciones privadas integradas por personas físicas o jurídicas que tengan por objeto la promoción de una o varias modalidades deportivas, la práctica de las mismas por sus asociados, así como la participación en actividades y competiciones deportivas». Uno de los aspectos más destacados de la naturaleza jurídica de los clubes deportivos es su condición de «entidad sin ánimo de lucro», circunstancia que le otorga un status peculiar, mediante el cual se desenvuelven en un entorno compartido con el sector público y el privado como un proveedor más de servicios deportivos para la comunidad. Puig, Domínguez y López (1996) destacan su diversidad social y su estrecha relación con el entramado institucional, centrando su función principal en la organización de equipos que participen en competiciones, pero no debe olvidarse que hoy en día son proveedores de primer orden de servicios de ocio, deporte y programas sociales (Wicker y Breuer, 2015), muy sensibles al entorno político, social y económico.

Se registra un aumento del $5,54 \%$ en el número de clubes deportivos desde 2012, pasando de 62.346 clubes deportivos federados (MECD, 2013, p.95) a 66.004 en el año 2016. Este ligero aumento también se ha visto reflejado en las licencias federativas, ya que en 2012 se registraron 3.499.000, un 2,43\% menos que en 2016, año en el que se registraron 3.586 .133 , y un $6,98 \%$ menos que en 2017 que se registraron 3.761.498 (MECD, 2018, p.110).

El club deportivo suele ser de tamaño medio o pequeño, teniendo una gran movilidad los deportistas que lo componen (Thiel y Mayer, 2009). Se caracteriza por una motivación intrínseca tanto por parte del personal interno del club como de los propios deportistas, mientras que la actividad principal del club está centrada en la organización de equipos -normalmente unideportivosque participan en competiciones a diferentes niveles (local, autonómico y nacional) (Puig et al., 1996).

Una de las peculiaridades con las que han convivido históricamente los clubes deportivos al amparo de su condición de entidad sin ánimo de lucro, no es otra que la ambigüedad en la relación existente entre la entidad y el personal que presta en ella sus servicios: $¿$ asalariados o voluntarios? La encrucijada de la profesionalización ha estado siempre en debate. Según Pereira (2016), la realidad actual muestra la necesaria profesionalización, ya no solo en la oferta de servicios sino también en la gestión de la entidad. Dicho autor defiende que este hecho, necesario a la par que obligatorio, ya comienza a producirse.

Existe una elevada sensibilidad al tema de la profesionalización: sirva de ejemplo lo recogido por Garavelli (2013), cuando anunció una «campaña de inspecciones del Ministerio de Trabajo, que aspira a 
que las entidades deportivas den de alta en la Seguridad Social a cualquier empleado que tenga una prestación económica», señalando que esta medida ponía seriamente en peligro el tejido deportivo asociativo, ya bastante castigado por importantes reducciones de las ayudas públicas recibidas.

Por otra parte, además de los clubes deportivos, que representan en mayor parte al tejido asociativo, estarían las uniones deportivas que tienen una función menos deportivizada, ya que normalmente no tienen como finalidad la competición, sino únicamente el ocio y la recreación son sus intenciones prioritarias.

Además de las anteriores, también podríamos encontrar las asociaciones deportivas, relacionadas en la mayor parte de los casos, con la promoción del deporte a toda la población, centrándose, en ocasiones en personas con necesidades especiales o en riesgo de exclusión social. Se caracterizan por no contar con instalaciones propias y debido a su importancia social es factible el establecimiento de diferentes convenios de colaboración con ayuntamientos u organizaciones sin ánimo de lucro.

\section{Ámbito deportivo privado}

Basándonos en Martínez-Lemos y Romo-Pérez (2015), las entidades deportivas privadas se pueden dividir en (i) organizaciones privadas de naturaleza asociativa y (ii) organizaciones privadas de naturaleza mercantil.

Dentro de las primeras -naturaleza asociativa- estaría el Comité Olímpico Español (COE) definido por el Consejo Superior de Deportes (CSD, 2017) como una asociación sin fines de lucro, dotada de personalidad jurídica y declarada de utilidad pública, cuyo objeto es el desarrollo olímpico y la difusión de los ideales olímpicos, así como el Comité Paralímpico Español (CPE). Se rige por sus propios reglamentos y estatutos y tiene diferentes comisiones, dentro de las cuales se pueden encontrar: marketing, financiación y gestión federativa o sostenibilidad, cooperación e integración, entre otras.

Según el CSD, el COE tiene como funciones principales la difusión de los valores olímpicos tanto a nivel escolar como universitario, así como la colaboración y coordinación con federaciones y cualquier entidad pública o privada que promueva el olimpismo.

Dentro de este tipo de organizaciones privadas encontraríamos también las conocidas federaciones deportivas españolas y las federaciones deportivas autonómicas. Las federaciones deportivas españolas, son definidas por Ley 10/1990, del 15 de octubre, del Deporte como: (...) entidades asociativas privadas, sin ánimo de lucro y con personalidad jurídica y patrimonio propio e independiente del de sus asociados. Además de sus propias atribuciones, ejercen por delegación funciones públicas de carácter administrativo, actuando en este caso, como agentes colaboradores de la Administración pública.

Están integradas por las Federaciones deportivas autonómicas y diferentes entidades que fomentan la promoción y el desarrollo de una modalidad deportiva concreta. En la actualidad existen en España 59 Federaciones, de las cuales 35 son federaciones olímpicas y 24 federaciones no olímpicas. Estas tienen sus funciones de organización, gestión y normalización de cada una de las especialidades de la modalidad deportiva. Dentro de las funciones de carácter administrativo destacaríamos la coordinación con las federaciones autonómicas, apoyo a los deportistas de élite, así como gestionar las subvenciones y los temas de dopaje, entre otras.

Por otro lado, dentro de las organizaciones privadas de naturaleza mercantil encontraríamos las empresas privadas de servicios deportivos. El deporte y las empresas deportivas tienen una increíble capacidad de movilización y convocatoria, por lo que han adquirido progresivamente un protagonismo, que hace que se haya estudiado mucho en los últimos años. Se sabe que en torno al deporte existe una importante actividad económica, pero no es fácil medir y cuantificar cuán fuerte es esa aportación (Rochel y Arias, 2012). Todos conocemos que absorbe una importante porción del ocio, pero son muchas las influencias que tiene en diferentes ámbitos difíciles de cuantificar como por ejemplo: los movimientos de apuestas deportivas o juegos de azar, el consumo de bebidas isotónicas, o el turismo deportivo, por poner varios ejemplos.

El Anuario de Estadísticas Deportivas (MECD, 2018, p. 51) revela que el número de empresas recogidas en el Directorio Central de Empresas (DIRCE) cuya actividad económica principal es deportiva, ascendió a 34.203 en 2017. En estas sociedades se han incluido empresas de actividades deportivas (81\%), de fabricación de artículos de deporte $(1 \%)$ y comercio al por menor de artículos deportivos (18\%). No obstante, cabe destacar la tendencia creciente observada en los últimos años, donde, a pesar 
de la coyuntura económica negativa, ya comentada en anteriores ocasiones, el número de empresas de este sector no ha dejado de crecer desde 2008, teniendo entre 2015 y 2016 un crecimiento del 5,84\%.

\section{Discusión y Conclusiones}

Las organizaciones necesitan una diferenciación y crear una ventaja competitiva debido a la mayor exigencia por parte de los clientes y usuarios y la creciente competencia en los mercados. En la línea de lo expuesto por GrimaldiPuyana y Ferrer (2016), se puede decir que este constante cambio obliga a las entidades a estar en continuo aprendizaje y reciclaje, tanto de los servicios ofertados como de la gestión de los mismos. En el contexto de recesión económica en el que nos encontramos, los presupuestos públicos destinados al deporte cada vez son más ajustados, como se puede observar en el Anuario de Estadísticas Deportivas (2017). Este hecho empuja a las administraciones a barajar otras opciones de gestión en busca de la oferta de servicios que merece la población y la sostenibilidad, que es cada vez más difícil de conseguir.

Existen tres ámbitos diferentes -público, privado y asociativo- que tienen sus propias características, pero hay que destacar que todos ellos buscan una promoción y fomento de la actividad física (Pereira, 2016). El ámbito público aboga más por una oferta de servicios pensando en toda la población. Se han comentado diferentes formas de gestión pública, ya que debido a la externalización de los servicios hay múltiples variantes, no recogidas todas en este estudio.

Por otro lado, el ámbito privado se centra más en una diferenciación del resto de organizaciones, ya que no tiene como objetivo prioritario las actividades para todo tipo de público, sino que en muchos casos son más especializadas, como pueden ser también las federaciones deportivas o el Comité Olímpico Español (COE).

Por último, el tejido asociativo en España tiene un gran peso, ya que oferta todo tipo de actividades para la población general. La estructura jurídica y funcional es diversa pero su característica de sin ánimo de lucro, le aporta un valor añadido, respecto a las demás. Dependiendo si es club deportivo, o asociación, tendrá una finalidad más competitiva o recreativa. Cabe destacar que este ámbito está en crecimiento, en contraste con el detrimento de la gestión municipal tradicional (Rodríguez et al., 2018).
Merece mención en el presente estudio la importancia de la innovación y el emprendimiento en la gestión de organizaciones deportivas, así como la especialización de los gestores deportivos, ya que deriva en una mejora consecuente de los procesos, técnicas y herramienta de la gestión deportiva. Como ya se ha dicho anteriormente, nos encontramos en una sociedad cambiante $y$ orientada al progreso, por lo que no puede tener lugar un estancamiento en el ámbito deportivo. El futuro de la gestión deportiva no es concreto, las demandas de la población seguirán cambiando a un ritmo acelerado, con ello, las adaptaciones de las entidades que ofertan los servicios y, en consecuencia, la tendencia en sus modelos estructurales, organizativos y deportivos (Pereira, 2016).

Por todo lo anterior, es importante basarnos en literatura previa para conocer más las prioridades que tienen los usuarios de las instalaciones deportivas y cómo son estas, en función de diferentes variables, como el precio (Calabuig, Núñez-Pomar, Prado-Gascó y Añó, 2014), la percepción de marca y comportamiento del consumidor (Alguacil, Alonso-Dos Santos y Pastor-Barceló, 2016) e incluso analizando el usuario interno de las organizaciones deportivas y su clima organizacional, dependiendo del tipo de organizaciones deportivas (Escamilla-Fajardo, Núñez-Pomar y Gómez-Tafalla, 2016).

Las implicaciones prácticas que puede tener este trabajo pueden ser diversas. Conocer los tipos de organizaciones deportivas en España, puede ayudar en diferentes estudios que engloben organizaciones deportivas a basarse en los ámbitos que más interesen. El presente estudio es una breve síntesis de los tipos de organizaciones existentes, por lo que puede ser de interés para crear una visión general a quien tiene un desconocimiento de la situación deportiva española.

Como futuras líneas de investigación, una importante propuesta sería realizar una revisión bibliográfica en la que se caractericen de forma más exhaustiva a las organizaciones deportivas españolas. Además, sería interesante hacer una investigación con una muestra representativa de organizaciones en cada ámbito de actuación. De este modo se podría definir, así como relacionar con diferentes variables propias de las organizaciones. 


\section{Referencias}

1. Alguacil, M., Dos Santos, M. A., Pastor-Barceló, A., y Colino, O. (2016). Análisis cualitativo comparativo de la lealtad en servicios públicos deportivos. Revista de psicología del deporte, 25(1), 69-72.

2. Arboledas, D.,y Puig, N. (2012). Análisiscomparativo de los servicios deportivos municipales de Andalucía y Cataluña. RICYDE. Revista Internacional de Ciencias del Deporte, 8(29), 223-244. doi: http:// dx.doi.org/10.5232/ricyde2012.02903

3. Calabuig, F., Núñez-Pomar, J., Prado-Gascó, V., y Añó, V. (2014). Effect of price increases on future intentions of sport consumers. Journal of business research, 67(5), 729-733.

4. Calabuig-Moreno, F., Quintanilla-Pardo, I., y Mundina-Gómez, J. (2008). La calidad percibida de los servicios deportivos: diferencias según instalación, género, edad y tipo de usuario en servicios náuticos. RICYDE. Revista Internacional de Ciencias del Deporte, 4(10), 25-43. doi: https:// doi.org/10.5232/ricyde2008.01003

5. Conde-Ruiz, J. I., Díaz, M., y Marín, C. (2016). Evolución del Gasto Público por Funciones durante la crisis (2007-2014): España vs UE. Documento de Trabajo, 09.

6. Escamilla-Fajardo, P., Núñez-Pomar, J. M., y Gómez-Tafalla, A. M. (2016). Clima Organizacional y sector de pertenencia: un análisis de la percepción de los empleados de entidades deportivas. Revista de psicología del deporte, 25(1), 73-76.

7. Fernández, J. E. R., Couto, J. M. P., y Aguilar, J. P. (2015). Análisis de la promoción deportiva en el municipio de Boiro (A Coruña): Relación entre la gestión deportiva municipal, los clubes deportivos y los resultados deportivos. EmásF: Revista digital de educación física, 6(33), 66-86.

8. Gallardo, L., Guerrero, L. G., y Gómez, A. J. (2004). La gestión de los servicios deportivos municipales: vías para la excelencia. Inde publicaciones. Barcelona: España.

9. García Ferrando, M., y Llopis Goig, R. (2017). La popularización del deporte en España: encuestas de hábitos deportivos 1980-2015. CIS-Centro de Investigaciones Sociológicas - Consejo Superior de
Deportes. Madrid: España.

10. Grimaldi Puyana, M., y Ferrer Cano, P. (2016). Dimensión y perfil de las organizaciones de gestión de instalaciones deportivas. Apunts: Educación física $y$ deportes, 4(126), 72-78.

11. Leiva, A. P., Márquez, P. G.-O., Rodríguez, C. G., Navas, J. M. M., y Bozal, R. G. (2017). Ejercicio físico y calidad de vida en estudiantes universitarios. International Journal of Developmental and Educational Psychology, 2(1), 437-446.

12. Martínez-Lemos, I., y Romo-Pérez, V. (2015). El sector privado del deporte en España: Relación con población, producción y renta. Retos, 28, 71-77.

13. Pereira, E. B. (2016). Tendencias en gestión deportiva. Revista española de educación física y deportes, 3(414), 13-16.

14. Puig, N., Domínguez, A. M., y López, C. (1996). Propuesta de marco teórico interpretativo sobre el asociacionismo deportivo en España. European Journal of Human Movement, 1(2), 75-92.

15. Grimaldi, M., Fernández, J., y García-Fernández, J. (2013). Situación laboral de gestores deportivos en servicios públicos externalizados. Actividad física $y$ deporte: ciencia y profesión, 1(18), 33-40.

16. Quesada, J. L. (2014). El control municipal de las concesiones administrativas: el caso de los servicios e instalaciones deportivas. Auditoría Pública, 62, 63-81.

17. Rochel, J. J. B., y Arias, B. L. (2012). Los avances en la valoración económica del deporte en Europa. Estudios de Economía Aplicada, 30(2), 637-654

18. Rodríguez, C. J. L., Calvo, B. L., Caba, M. M. G., Sánchez, M. J., Casado, J. E., Feito, T. E., y Lis, F. B. (2018). Asociacionismo deportivo: diagnóstico y perspectivas. Editorial Reus. Madrid: España

19. 19.Salgado-Barandela, J., Barajas, A., y SánchezFernández, P. (2017). Impacto económico del deporte: tema de interés creciente en la literatura científica. Revista Internacional de Medicina $y$ Ciencias de la Actividad Física y del Deporte, 17(68), 729-755. doi: https://doi.org/10.15366/ rimcafd2017.68.010

20. Sanz, R. P., y Insúa, J. A. S. (2003). El impacto del deporte en la economía: problemas de medición. 
RAE: Revista Asturiana de Economía, 26, 61-84.

21. Thiel, A., y Mayer, J. (2009). Characteristics of voluntary sports clubs management: A sociological perspective. European sport management quarterly, 9(1), 81-98.

22. Wicker, P., y Breuer, C. (2015). How the economic and financial situation of the community affects sport clubs' resources: Evidence from multi-level models. International Journal of Financial Studies, 3(1), 31-48. 